\title{
Dispersive and chiral symmetry constraints on the light meson form factors
}

\author{
Irinel Caprini \\ National Institute of Physics and Nuclear Engineering, \\ POB MG 6, Bucharest, R-76900 Romania
}

\begin{abstract}
The form factors of the light pseudoscalar mesons are investigated in a dispersive formalism based on hadronic unitarity, analyticity and the OPE expansion of the QCD Green functions. We propose generalizations of the original mathematical techniques, suitable for including additional low energy information provided by experiment or Chiral Perturbation Theory (CHPT). The simultaneous treatment of the electroweak form factors of the $\pi$ and $K$ mesons allows us to test the consistency with QCD of a low energy CHPT theorem. By applying the formalism to the pion electromagnetic form factor, we derive quite strong constraints on the higher Taylor coefficients at zero momentum, using information about the phase and the modulus of the form factor along a part of the unitarity cut.
\end{abstract}




\section{Introduction}

Chiral perturbation theory [1] provides a systematic low energy expansion of the QCD Green functions in the sector of light mesons. Calculations up to two loops were done recently for the scattering amplitudes and the electroweak form factors in the chiral $S U(2) \times S U(2)$ limit [2]-[5]. A full $\mathcal{O}\left(p^{6}\right) S U(3) \times S U(3)$ calculation was also performed [6] for a specific combination of form factors which does not involve arbitrary renormalization constants. The result allowed to estimate the symmetry breaking corrections to a low energy theorem proposed by Sirlin [7], which generalizes the Ademollo-Gatto theorem [8].

The fundamental properties of causality and unitarity are important ingredients in CHPT. Dispersion relations for scattering amplitudes and form factors or their inverses were used in performing low order calculations [2], [5], or as a method of effective resummation of the high order terms [9], [10]. In the present paper we shall consider an alternative dispersive method suitable especially for form factors [11],[12], which uses as input an information about the modulus along the unitarity cut, leading to constraints on the values inside the analyticity domain. The technique was for the first time combined with the OPE expansion of a QCD polarization function in [13]. Recently, this formalism was successfully applied to the form factors describing the weak semileptonic decays of heavy mesons [14]-[21]. Quite strong constraints on the shape of these form factors near zero recoil have been obtained by combining the dispersive formalism with the predictions of the heavy quark effective theory (HQET) [22], [23]. The predictive power of the technique was considerably increased [17], [20], [21] by the simultaneous treatment of several form factors with different unitarity thresholds, connected among them near zero recoil by HQET.

It is of interest to apply this formalism to the form factors of the light pseudoscalar mesons, for which chiral symmetry predicts definite correlations for certain kinematical points. A first investigation of the weak form factors of the $K \rightarrow \pi l \nu$ decay in this framework was performed in [13]. Similar techniques were applied in [24] in order to parametrize the modulus of the pion electromagnetic form factor in the time like region. In the present paper we perform an analysis of the electromagnetic and weak form factors of the $\pi$ and $K$ mesons, with emphasis on their low energy expansions. The purpose of the investigation is to see whether the dispersive approach is useful for testing the rigorous predictions of chiral symmetry and for constraining the free parameters of CHPT. To this end we present generalizations of the standard technique suitable for incorporating additional information provided by experiments or CHPT.

The paper is organized as follows. In the next Section we review the dispersive formalism: first we present the form factors of interest and some of their properties, then we describe the physical input of the method and the standard mathematical techniques used for optimally exploiting this input. In Section 3 we illustrate the simultaneous treatment of several form factors with different unitarity branch points by performing a test of a low energy theorem of CHPT. In Section 4 we derive constraints on the Taylor coefficients at zero momentum of the pion electromagnetic form factor. We first give the simple unitar- 
ity bounds obtained from the standard formalism and then show how they are improved using additional information on the phase and the modulus of the form factor along a part of the unitarity cut. In the last Section we present some conclusions.

\section{Review of the dispersive formalism}

\subsection{Definitions and notations}

We consider the electromagnetic form factors of the $\pi$ and $K$ mesons, defined as

$$
\begin{array}{r}
\left\langle\pi^{+}\left(p^{\prime}\right)\left|J_{\mu}^{e l m}\right| \pi^{+}(p)\right\rangle=\left(p+p^{\prime}\right)_{\mu} F_{\pi}\left(q^{2}\right) \\
\left\langle K^{+}\left(p^{\prime}\right)\left|J_{\mu}^{e l m}\right| K^{+}(p)\right\rangle=\left(p+p^{\prime}\right)_{\mu} F_{K^{+}}\left(q^{2}\right) \\
\left\langle K^{0}\left(p^{\prime}\right)\left|J_{\mu}^{\text {elm }}\right| K^{0}(p)\right\rangle=\left(p+p^{\prime}\right)_{\mu} F_{K^{0}}\left(q^{2}\right),
\end{array}
$$

and the form factors describing the weak semileptonic decay $K \rightarrow \pi l \nu$ :

$$
\begin{aligned}
\left\langle\pi^{0}\left(p^{\prime}\right)\left|J_{\mu}^{w}\right| K^{+}(p)\right\rangle & =\left(p+p^{\prime}\right)_{\mu} f_{\pi K}^{(+)}\left(q^{2}\right)+\left(p-p^{\prime}\right)_{\mu} f_{\pi K}^{(-)}\left(q^{2}\right) \\
& =\left(q_{\mu}-\frac{q \cdot p}{q^{2}} P_{\mu}\right) f_{\pi k}\left(q^{2}\right)+q_{\mu} d_{\pi k}\left(q^{2}\right),
\end{aligned}
$$

where $q=p-p^{\prime}, P=p+p^{\prime}$ and $J_{\mu}^{e l m}\left(J_{\mu}^{w}\right)$ is the electromagnetic (weak) current:

$$
J_{\mu}^{e l m}=\frac{2}{3} \bar{u} \gamma_{\mu} u-\frac{1}{3} \bar{d} \gamma_{\mu} d-\frac{1}{3} \bar{s} \gamma_{\mu} s, \quad J_{\mu}^{w}=\bar{s} \gamma_{\mu} u .
$$

The form factors defined in (1) and (2) are analytic functions of real type in the complex plane $t=q^{2}$ cut along the real axis from the threshold of two particle production to infinity. The cut starts at $t_{\pi}=4 m_{\pi}^{2}$ for the electromagnetic form factors $F_{\pi}(t), F_{K^{+}}(t)$ and $F_{K^{0}}(t)$, and at $t_{\pi K}=\left(m_{\pi}+m_{K}\right)^{2}$ in the case of the weak form factors $d_{\pi K}(t)$ and $f_{\pi K}(t)$. At $t=0$ the conservation of the electromagnetic current and the Ademollo-Gatto theorem [8] give

$$
\begin{array}{r}
F_{\pi}(0)=1, F_{K^{+}}(0)=1, F_{K^{0}}(0)=0, \\
d_{\pi K}(0)=\left(m_{K}^{2}-m_{\pi}^{2}\right) f_{\pi K}(0), \quad f_{\pi K}(0)=1 .
\end{array}
$$

If we define the function

$$
\Delta(t)=\frac{1}{2} F_{\pi}(t)+\frac{1}{2} F_{K^{+}}(t)+F_{K^{0}}(t)-f_{K \pi}(t),
$$

from (4) it follows that

$$
\Delta(0)=0 .
$$

This relation is valid up to terms quadratic in the chiral symmetry breaking parameters [8]. The Ademollo-Gatto theorem was extended by Sirlin [7] to values $t \neq 0$ near the origin. 
Sirlin's theorem requires in particular the vanishing of the derivatives of the function $\Delta(t)$ at $t=0$ :

$$
\Delta^{\prime}(0)=0, \quad \Delta^{\prime \prime}(0)=0, \ldots
$$

In CHPT the renormalization constants cancel in the combination of the form factors entering Sirlin's function. A recent $\mathcal{O}\left(p^{6}\right)$ calculation in full chiral $S U(3) \times S U(3)$ perturbation theory gave the value [6]

$$
\Delta^{\prime}(0)=\frac{1}{6} r_{S}^{2}=(0.0033 \pm 0.0005) \mathrm{fm}^{2},
$$

where $r_{S}^{2}$ is the "charge radius" of the Sirlin's form factor [6]. This result shows explicitely the breaking symmetry correction to Sirlin theorem (7).

\subsection{Unitarity and dispersion inequalities}

Dispersive bounds on the above form factors are obtained by considering the vacuum polarization tensor :

$$
i \int d x e^{i q x}\left\langle 0\left|T\left(J_{\mu}^{\dagger}(x) J_{\nu}(0)\right)\right| 0\right\rangle=\left(q_{\mu} q_{\nu}-g_{\mu \nu} q^{2}\right) \Pi\left(q^{2}\right)+g_{\mu \nu} D\left(q^{2}\right),
$$

where $J_{\mu}$ denotes either the electromagnetic $J_{\mu}^{e l m}$ or the weak $J_{\mu}^{w}$ current (the function $D\left(q^{2}\right)$ vanishes in the electromagnetic case). From the asymptotic behaviour of QCD it follows that the derivative $\Pi^{\prime}\left(q^{2}\right)$ of the amplitude $\Pi\left(q^{2}\right)$ satisfies the dispersion relation

$$
\Pi^{\prime}\left(q^{2}\right)=\frac{1}{\pi} \int_{0}^{\infty} \frac{\operatorname{Im} \Pi(t+i \epsilon)}{\left(t-q^{2}\right)^{2}} \mathrm{~d} t,
$$

with the spectral function $\operatorname{Im} \Pi(t+i \epsilon)$ given by hadronic unitarity. Using the definitions (1) of the electromagnetic form factors and taking into account the positivity $\operatorname{Im} \Pi(t+i \epsilon) \geq 0$, we obtain the inequality:

$$
\begin{gathered}
\operatorname{Im} \Pi_{e l m}(t+i \epsilon) \geq \frac{1}{48 \pi}\left(1-\frac{t_{\pi}}{t}\right)^{3 / 2}\left|F_{\pi}(t)\right|^{2} \theta\left(t-t_{\pi}\right) \\
+\frac{1}{48 \pi}\left(1-\frac{t_{K}}{t}\right)^{3 / 2}\left[\left|F_{K^{+}}(t)\right|^{2}+\left|F_{K^{0}}(t)\right|^{2}\right] \theta\left(t-t_{K}\right),
\end{gathered}
$$

where $t_{\pi}=4 m_{\pi}^{2}$ and $t_{K}=4 m_{K}^{2}$ are unitarity branch points. By inserting the inequality (11) in the dispersion relation (10) for an euclidian point $q^{2}=-Q^{2}<0$ we obtain:

$$
\begin{gathered}
\Pi_{\text {elm }}^{\prime}\left(-Q^{2}\right) \geq \frac{1}{48 \pi^{2}} \int_{t_{\pi}}^{\infty} \frac{\mathrm{d} t}{\left(t+Q^{2}\right)^{2}}\left(1-\frac{4 m_{\pi}^{2}}{t}\right)^{3 / 2}\left|F_{\pi}(t)\right|^{2} \\
+\frac{1}{48 \pi^{2}} \int_{t_{K}}^{\infty} \frac{\mathrm{d} t}{\left(t+Q^{2}\right)^{2}}\left(1-\frac{t_{K}}{t}\right)^{3 / 2}\left[\left|F_{K^{+}}(t)\right|^{2}+\left|F_{K^{0}}(t)\right|^{2}\right] .
\end{gathered}
$$


A relation similar to (12) can be written for the polarization function $\Pi_{w}\left(Q^{2}\right)$ of the weak current. Using the definition (2) we obtain from unitarity and positivity

$$
\begin{aligned}
& \operatorname{Im} \Pi_{w}(t+i \epsilon) \geq \frac{\eta}{48 \pi}\left(1-\frac{t_{\pi K}}{t}\right)^{1 / 2}\left(1-\frac{t_{\pi K}^{(-)}}{t}\right)^{1 / 2} \times \\
& {\left[\left(1-\frac{t_{\pi K}}{t}\right)\left(1-\frac{t_{\pi K}^{(-)}}{t}\right)\left|f_{\pi K}\right|^{2}+\frac{\left|d_{\pi K}\right|^{2}}{t^{2}}\right] \theta\left(t-t_{\pi K}\right),}
\end{aligned}
$$

where $t_{\pi K}=\left(m_{K}+m_{\pi}\right)^{2}, t_{\pi K}^{(-)}=\left(m_{K}-m_{\pi}\right)^{2}$ and $\eta=\frac{3}{2}$ is an isospin factor [13]. By inserting (13) in a dispersion relation of the form (10) we obtain

$$
\begin{aligned}
& \Pi_{w}^{\prime}\left(-Q^{2}\right) \geq \frac{\eta}{48 \pi^{2}} \int_{t_{\pi K}}^{\infty} \frac{\mathrm{d} t}{\left(t+Q^{2}\right)^{2}}\left(1-\frac{t_{\pi K}}{t}\right)^{1 / 2}\left(1-\frac{t_{\pi K}^{(-)}}{t}\right)^{1 / 2} \\
& \times\left[\left(1-\frac{t_{\pi K}}{t}\right)\left(1-\frac{t_{\pi K}^{(-)}}{t}\right)\left|f_{\pi K}(t)\right|^{2}+\frac{\left|d_{\pi K}(t)\right|^{2}}{t^{2}}\right] .
\end{aligned}
$$

It is interesting to note that the inequalities (12) and (14) can be added, leading to a single inequality

$$
\begin{aligned}
& \Pi^{\prime}\left(-Q^{2}\right) \geq \frac{1}{48 \pi^{2}} \int_{t_{\pi}}^{\infty} \frac{\mathrm{d} t}{\left(t+Q^{2}\right)^{2}}\left(1-\frac{4 m_{\pi}^{2}}{t}\right)^{3 / 2}\left|F_{\pi^{+}}(t)\right|^{2} \\
& +\frac{1}{48 \pi^{2}} \int_{t_{K}}^{\infty} \frac{\mathrm{d} t}{\left(t+Q^{2}\right)^{2}}\left(1-\frac{t_{K}}{t}\right)^{3 / 2}\left[\left|F_{K^{+}}(t)\right|^{2}+\left|F_{K^{0}}(t)\right|^{2}\right] \\
& +\frac{\eta}{48 \pi^{2}} \int_{t_{\pi K}}^{\infty} \frac{\mathrm{d} t}{\left(t+Q^{2}\right)^{2}}\left(1-\frac{t_{\pi K}}{t}\right)^{1 / 2}\left(1-\frac{t_{\pi K}^{(-)}}{t}\right)^{1 / 2} \\
& \quad \times\left[\left(1-\frac{t_{\pi K}}{t}\right)\left(1-\frac{t_{\pi K}^{(-)}}{t}\right)\left|f_{\pi K}(t)\right|^{2}+\frac{\left|d_{\pi K}(t)\right|^{2}}{t^{2}}\right],
\end{aligned}
$$

where

$$
\Pi^{\prime}\left(-Q^{2}\right)=\Pi_{e l m}^{\prime}\left(-Q^{2}\right)+\Pi_{w}^{\prime}\left(-Q^{2}\right) .
$$

In the euclidian region $Q^{2}>0$, the functions $\Pi_{\text {elm }}^{\prime}\left(-Q^{2}\right)$ and $\Pi_{w}^{\prime}\left(-Q^{2}\right)$ can be calculated by applying renormalization group improved perturbative QCD, with nonperturbative corrections included by means of operator product expansions (OPE). We used the expressions given in [25]:

$$
\begin{aligned}
& \Pi^{\prime}\left(-Q^{2}\right)= \\
& =\left(\frac{1}{6}+\frac{1}{4}\right) \frac{1}{\pi^{2} Q^{2}}\left[1+\frac{\alpha_{s}\left(-Q^{2}\right)}{\pi}+F_{3}\left(\frac{\alpha_{s}\left(-Q^{2}\right)}{\pi}\right)^{2}+\left(F_{4}+\frac{\beta_{1}^{2} \pi^{2}}{12}\right)\left(\frac{\alpha_{s}\left(-Q^{2}\right)}{\pi}\right)^{3}\right] \\
& -\left(\frac{1}{6}+\frac{3}{4}\right) \frac{1}{\pi^{2} Q^{4}} m_{s}^{2}\left(-Q^{2}\right)+\left(\frac{1}{18}+\frac{1}{12}\right) \frac{1}{Q^{6}}\left\langle\frac{\alpha_{s}}{\pi} \bar{G} G\right\rangle+\left(\frac{10}{9}+1\right) \frac{1}{Q^{6}} m_{q}\langle\bar{\psi} \psi\rangle,
\end{aligned}
$$


corresponding to $n_{f}=3$ flavours. The first and the second numerical constant in front of each term indicate the separate contribution of $\Pi_{\text {elm }}^{\prime}\left(-Q^{2}\right)$ and $\Pi_{w}^{\prime}\left(-Q^{2}\right)$, respectively. To evaluate these quantities we used the two-loop expressions of the running coupling $\alpha_{s}\left(-Q^{2}\right)$ and the running mass $m_{s}\left(-Q^{2}\right)$ of the $s$-quark [25], the perturbative parameters $F_{3}=1.6398, F_{4}=-10.2839, \beta_{1}=-9 / 2[25]$ and the condensates $m_{q}\langle\bar{\psi} \psi\rangle=(0.200 \mathrm{GeV})^{4}$ and $\left\langle\alpha_{s} \bar{G} G\right\rangle / \pi=(0.45 \mathrm{GeV})^{4}$ [26]. As concerns the value of the euclidian point $Q^{2}$ at which we calculate the polarization amplitude, it must be on one hand high enough to ensure the validity of the OPE expansion, and on the other hand small enough to provide a strong constraint on the form factors through the dispersion relation (10). In fact, the results turn out to be rather stable when $Q^{2}$ is varied in the range $2 \mathrm{GeV}^{2} \leq Q^{2} \leq 6 \mathrm{GeV}^{2}$. The results reported in this work are obtained with the choice $Q^{2}=2 \mathrm{GeV}^{2}$, for which $\Pi_{\text {elm }}^{\prime}\left(-Q^{2}\right)=0.009546 \mathrm{GeV}^{-2}$ and $\Pi_{w}^{\prime}\left(-Q^{2}\right)=0.0133 \mathrm{GeV}^{-2}$. With the l.h.s. of the relations (12), (14) or (15) known, these inequalities are integral conditions for the sum of the moduli squared of the corresponding form factors.

It is useful to point out that an alternative integral condition for the electromagnetic form factors can be obtained by using as input, instead of OPE in the euclidian region, a lower bound on the hadronic muon anomaly. This method was first proposed in [27], and starts from the the vacuum polarization contribution of the hadronic part of the muon anomaly

$$
a_{\mu}^{(h)}=\frac{1}{\pi} \int_{0}^{\infty} \frac{\mathrm{d} t}{t} \operatorname{Im} \Pi(t+i \epsilon) \mathcal{K}(t),
$$

where

$$
\mathcal{K}(t)=\frac{\alpha}{\pi} \int_{0}^{1} \frac{z^{2}(1-z)}{z^{2}+(1-z) t / m_{\mu}^{2}} \mathrm{~d} z .
$$

By introducing (18) in the unitarity relation (11) we obtain the inequality

$$
\begin{array}{r}
a_{\mu}^{(h)} \geq \frac{1}{48 \pi^{2}} \int_{t_{\pi}}^{\infty} \frac{K(t)}{t}\left(1-\frac{4 m_{\pi}^{2}}{t}\right)^{3 / 2}\left|F_{\pi^{+}}(t)\right|^{2} \mathrm{~d} t \\
+\frac{1}{48 \pi^{2}} \int_{t_{K}}^{\infty} \frac{K(t)}{t}\left(1-\frac{4 m_{K}^{2}}{t}\right)^{3 / 2}\left[\left|F_{K^{+}}(t)\right|^{2}+\left|F_{K^{0}}(t)\right|^{2}\right] \mathrm{d} t,
\end{array}
$$

which restricts the electromagnetic form factors along the unitarity cut. In the calculations we adopted the conservative lower bound $a_{\mu}^{(h)} \geq 7.5 \times 10^{-8}$ [28], based on the experimental value, the weak radiative corrections and an estimate of the hadronic box diagram.

\subsection{Standard mathematical techniques}

In this subsection we briefly review the standard techniques for exploiting in the optimal way the conditions (12), (14), (15) or (20) in order to obtain constraints on the form factors and their derivatives inside the analyticity domain. It is useful to introduce a 
compact notation, defining the form factors $F_{i}(t), i=1, . ., 5$ as

$$
\begin{aligned}
F_{1}(t)=F_{\pi}(t), & F_{2}(t)=F_{K^{+}}(t), F_{3}(t)=F_{K^{0}}(t) \\
F_{4}(t) & =f_{\pi K}(t), F_{5}(t)=d_{\pi K}(t),
\end{aligned}
$$

and denoting by $t_{i}$ the unitarity branch points:

$$
\begin{array}{r}
t_{1}=t_{\pi}, t_{2}=t_{K}, t_{3}=t_{K}, \\
t_{4}=t_{\pi K}, t_{5}=t_{\pi K} .
\end{array}
$$

We now apply to each integral with the lower limit $t_{i}$ appearing in the conditions (12), (14), (15) or (20) the following conformal mapping

$$
z(t)=\frac{\sqrt{t_{i}-t}-\sqrt{t_{i}}}{\sqrt{t_{i}-t}+\sqrt{t_{i}}}
$$

By this transformation the complex $t$ plane is mapped onto the unit disk in the complex plane $z$, such that $z(0)=0$ and the unitarity cut $t \geq t_{i}$ becomes the boundary $|z|=1$. For simplicity we denote $F_{i}(z)=F_{i}(t(z))$, where $t(z)$ is the inverse of the mapping $z(t)$. Then the conditions (12), (14), (15) or (20) can be written in the canonical form:

$$
\frac{1}{2 \pi} \sum_{i=1}^{I} \int_{0}^{2 \pi}\left|w_{i}(\zeta) F_{i}(\zeta)\right|^{2} \mathrm{~d} \theta \leq 1, \quad \zeta=\exp (i \theta)
$$

where $I=3$ or $I=5$. In Eq.(24) $w_{i}(z)$ are analytic functions without zeros inside the unit disk, their modulus square on the boundary are equal to the weight functions appearing in front of the form factors, multiplied by the Jacobian of the conformal mapping (23). In mathematical books these functions are called "outer functions" and are defined in terms of their modulus on the boundary as [29]

$$
w_{i}(z)=\exp \left[\frac{1}{2 \pi} \int_{0}^{2 \pi} \frac{\zeta+z}{\zeta-z} \ln \left|w_{i}(\zeta)\right| \mathrm{d} \theta\right], \quad \zeta=\exp (i \theta) .
$$

In our case the functions $w_{i}(z)$ have simple explicit expressions [13]-[21]. For the global condition (15) which involves all the form factors, the outer functions $w_{i}(z)$ are

$$
\begin{gathered}
w_{i}(z)=\frac{\left(1-d_{i}\right)^{2}}{16} \sqrt{\frac{1}{6 \pi t_{i} \Pi^{\prime}\left(-Q^{2}\right)} \frac{(1+z)^{2} \sqrt{1-z}}{\left(1-z d_{i}\right)^{2}}}, \quad i=1,2,3, \\
w_{4}(z)=\frac{\left(1-d_{4}\right)^{2}}{32\left(1-z_{-}\right)^{3 / 2}} \sqrt{\frac{\eta_{4}}{6 \pi t_{4} \Pi^{\prime}\left(-Q^{2}\right)}} \frac{(1+z)^{2} \sqrt{1-z}\left(1-z z_{-}\right)^{3 / 4}}{\left(1-z d_{4}\right)^{2}}, \\
w_{5}(z)=\frac{\left(1-d_{5}\right)^{2}}{32 t_{5}\left(1-z_{-}\right)^{5 / 2}} \sqrt{\frac{\eta_{5}}{2 \pi t_{5} \Pi^{\prime}\left(-Q^{2}\right)}} \frac{(1+z)(1-z)^{5 / 2}}{\left(1-z z_{-}\right)^{1 / 4}\left(1-z d_{5}\right)^{2}},
\end{gathered}
$$


where

$$
d_{i}=\frac{\sqrt{t_{i}+Q^{2}}-\sqrt{t_{i}}}{\sqrt{t_{i}+Q^{2}}+\sqrt{t_{i}}}, \quad i=1, . ., 5
$$

and

$$
z_{-}=\frac{\sqrt{t_{\pi K}-t_{\pi K}^{(-)}}-\sqrt{t_{\pi K}}}{\sqrt{t_{\pi K}-t_{\pi K}^{(-)}}+\sqrt{t_{\pi K}}} .
$$

For the alternative condition $(20)$ the functions $w_{i}(z)$ are replaced by :

$$
w_{i}(z)=\frac{1}{16} \sqrt{\frac{1}{6 \pi t_{i} a_{\mu}^{(h)}}}(1+z)^{2} \sqrt{1-z} w_{\mathcal{K}}(z), \quad i=1,2,3,
$$

where $w_{\mathcal{K}}(z)$ is an outer function defined as in (25) in terms of its modulus $\left|w_{\mathcal{K}}(\zeta)\right|=$ $\sqrt{\mathcal{K}(\zeta)}$ on the boundary.

The inequality (24) is a standard boundary condition in $L^{2}$ norm for the functions $F_{i}(z)$ [29], from which one obtains constraints on the size and shape of these functions at interior points. The results are expected to be stronger if one keeps in (24) the contributions of all the functions $F_{i}(z)$ and exploits in addition the correlations among them provided by symmetries at some kinematical points. This technique proved to be very useful in the case of the weak form factors of heavy mesons, using HQET near zero recoil. In principle, a difficulty in applying the formalism to the excited states is the presence of the unphysical cuts below the unitarity branch points. In the case of heavy mesons, the unphysical cuts are well approximated by a few narrow resonances of known masses, which can be treated by adequate techniques with no assumption about the residua [14]-[21]. As we shall see in the next Section, the situation is more complicated in the case of the light mesons.

\section{Test of a CHPT low energy theorem}

In applying the relation (24) we recall that the functions $F_{1}, F_{4}$ and $F_{5}$, i.e. the electromagnetic form factor of the pion and the weak form factors are analytic below their unitarity branch points, while the kaon electromagnetic form factors, denoted here as $F_{2}$ and $F_{3}$, have an unphysical cut along the region $t_{\pi}<t<t_{K}$, below the unitarity threshold of the $K \bar{K}$ production. In the variable $z$ this cut is placed inside the unit disk $|z|<1$, along the segment $-1<z<z_{0}$, where $z_{0}$ denotes the image of the two pion production threshold by the conformal transformation (23) for the kaon form factors:

$$
z_{0}=\frac{\sqrt{t_{K}-t_{\pi}}-\sqrt{t_{K}}}{\sqrt{t_{K}-t_{\pi}}+\sqrt{t_{K}}}
$$

The dispersive formalism can be applied to this case only if some assumptions about the unphysical cut are adopted. For instance, if the phase of the kaon form factors along the unphysical cut is supposed to be known, the problem can be treated by means of a suitable modification of the standard techniques. Then one can use the inequalities 
(12) or (15) to correlate the low energy expansions of the electromagnetic and weak form factors or to test some predictions of chiral symmetry.

In this Section we will illustrate the procedure by testing the consistency of the relations (7) proposed by Sirlin [7], with the requirements of unitarity and perturbative QCD introduced in the condition (15). We adopt for the phase of the kaon electromagnetic form factors a model similar to the one proposed in [30] for the pion form factor, and generalized to include a narrow $\omega$ resonance, with the $\mathrm{SU}(3)$ relation $C_{\rho}=3 C_{\omega}$ between the residua. We assumed therefore that the phases of the form factors $F_{K^{+}}(t+i \epsilon)$ and $F_{K^{0}}(t+i \epsilon)$ along the unphysical region are

$$
\delta_{F_{K^{+}}}(t)=\operatorname{arctg}\left[\frac{3 m_{\rho} \Gamma_{\rho}(t)}{4\left(m_{\rho}^{2}-t\right)}\right], \quad t_{\pi}<t<t_{K}
$$

and

$$
\delta_{F_{K^{0}}}(t)=\operatorname{arctg}\left[\frac{3 m_{\rho} \Gamma_{\rho}(t)}{2\left(m_{\rho}^{2}-t\right)}\right], \quad t_{\pi}<t<t_{K} .
$$

We used the $\rho$ width $\Gamma_{\rho}(t)$ given by the resonance chiral effective theory [31], [32]:

$$
\Gamma_{\rho}(t)=\frac{m_{\rho} t}{96 \pi f_{\pi}^{2}}\left(1-\frac{4 m_{\pi}^{2}}{t}\right)^{3 / 2},
$$

where $f_{\pi}=93.1 \mathrm{MeV}$ is the pion decay constant.

We shall use now the information on the phase in order to remove the unphysical cut of the kaon form factors. To this end we shall use a so-called Omnès function [33], which exactly compensates the known phase of the form factors along this part of the cut, leaving us with functions analytic below the unitarity threshold. In what follows we describe briefly the method. Since we treat all the form factors simultaneously it is convenient to introduce a collective notation, defining

$$
\begin{array}{r}
O_{i}(t)=1, \quad i=1,4,5, \\
O_{i}(t)=\exp \left[\frac{t}{\pi} \int_{t_{\pi}}^{\infty} \frac{\delta_{i}\left(t^{\prime}\right)}{t^{\prime}\left(t^{\prime}-t\right)} \mathrm{d} t^{\prime}\right], \quad i=2,3,
\end{array}
$$

where the functions $\delta_{i}(t)$ are defined as $\delta_{2}(t)=\delta_{F_{K^{+}}}(t), \delta_{3}(t)=\delta_{F_{K^{0}}}(t)$ for $t_{\pi} \leq t \leq t_{K}$, and are extended for $t>t_{K}$ as Lipschitz continuous functions [29]. Of course, there is a large arbitrariness in such an extension, but the results are independent of the particular values of $\delta_{i}(t)$ for $t>t_{K}$. The reason is that, being analytic and without zeros in the $t$-plane cut along $\left(t_{\pi}, \infty\right)$, and with regular values on the boundary, $O_{2}(t)$ and $O_{3}(t)$ are outer functions, so by multiplying a class of functions with them, the class is not changed [29]. By construction, the phase of $O_{i}(t+i \epsilon)$ coincides with $\delta_{i}(t)$. We also use the notation $O_{i}(z)$ to denote these functions in terms of the variable $z$ defined in (23). The functions $O_{i}(z)$ are analytic in $|z|<1$, excepted for a cut along the segment $\left(-1, z_{0}\right)$, where the phase of $O_{i}(z-i \epsilon)$ is equal to $\delta_{i}(t(z))$ (we recall that the conformal transformation $(23)$ 
maps the upper half $t$-plane onto the lower semidisk in the $z$-plane). We define the set of functions $f_{i}(z)$ as

$$
f_{i}(z)=F_{i}(z)\left[O_{i}(z)\right]^{-1}, \quad i=1, . ., 5,
$$

where $F_{i}(z)$ are the form factors defined in (21). From (36) it is easy to see that the complex phases of the form factors $F_{2}(z)$ and $F_{3}(z)$ along the cut $\left(-1, z_{0}\right)$ are compensated by the phases of the Omnès functions $O_{i}(z)$. Therefore, all the functions $f_{i}(z)$ are analytic of real type inside the unit disk $|z|<1$. By introducing (36) in the $L^{2}$ norm condition (24) we have

$$
\begin{array}{r}
\frac{1}{2 \pi} \sum_{i=1}^{5} \int_{0}^{2 \pi}\left|w_{i}(\zeta) O_{i}(\zeta) f_{i}(\zeta)\right|^{2} \mathrm{~d} \theta= \\
\frac{1}{2 \pi} \sum_{i=1}^{5} \int_{0}^{2 \pi}\left|w_{i}(\zeta) \omega_{i}(\zeta) f_{i}(\zeta)\right|^{2} \mathrm{~d} \theta \leq 1, \quad \zeta=\exp (i \theta),
\end{array}
$$

where $w_{i}(z)$ are the outer functions defined in (26) and (27), and $\omega_{i}(z)$ are additional outer functions which satisfy the relations

$$
\left|\omega_{i}(\zeta)\right|=\left|O_{i}(\zeta)\right|, \quad \zeta=\exp (i \theta), \quad i=1, \ldots 5
$$

They can be calculated in terms of their modulus on the boundary using the standard formula [29] given in (25), which is equivalent to

$$
\omega_{i}(t)=\exp \left[\frac{\sqrt{t_{K}-t}}{\pi} \int_{t_{K}}^{\infty} \frac{\ln \left|O_{i}\left(t^{\prime}\right)\right|}{\sqrt{t^{\prime}-t_{K}}\left(t^{\prime}-t\right)} \mathrm{d} t^{\prime}\right], \quad i=1, \ldots .5 .
$$

It is convenient to introduce the new functions $g_{i}(z)$ as

$$
g_{i}(z)=w_{i}(z) \omega_{i}(z) f_{i}(z)=\Omega_{i}(z) F_{i}(z), \quad i=1, . ., 5
$$

where

$$
\Omega_{i}(z)=w_{i}(z) \omega_{i}(z)\left[O_{i}(z)\right]^{-1}
$$

Now, in terms of $g_{i}(z)$ the inequality (37) takes the canonical form

$$
\frac{1}{2 \pi} \sum_{i=1}^{5} \int_{0}^{2 \pi}\left|g_{i}(\zeta)\right|^{2} \mathrm{~d} \theta \leq 1, \quad \zeta=\exp (i \theta)
$$

By construction the functions $g_{i}(z)$ are analytic inside the disk $|z|<1$ and can be expanded as power series

$$
g_{i}(z)=\sum_{n=0}^{\infty} c_{n, i} z^{n}, \quad i=1, . .5
$$


with real coefficients $c_{n, i}=c_{n, i}^{*}$. By inserting these expansions in (42) we obtain

$$
\sum_{i=1}^{5} \sum_{n=0}^{\infty} c_{n, i}^{2} \leq 1
$$

As we discussed in Section 2, the generalization of the Ademollo-Gatto theorem proposed by Sirlin requires the vanishing of the first derivatives at $t=0$ of the function $\Delta(t)$ defined in $(5)$, in the exact chiral limit. In order to test the consistency of this condition, let us put

$$
\Delta^{(k)}(0)=s_{k}, \quad k=1, \ldots . K,
$$

where the derivatives are with respect to $t$, and $s_{k}$ are given numbers of the order of magnitude of chiral symmetry breaking [6]. Using (40) we write $\Delta(t)$ as

$$
\Delta(t)=\sum_{i=1}^{5} a_{i}\left[\Omega_{i}(z)\right]^{-1} g_{i}(z)
$$

where

$$
a_{1}=\frac{1}{2}, a_{2}=\frac{1}{2}, a_{3}=1, a_{4}=-1, a_{5}=0 .
$$

Therefore the derivatives entering (45) become

$$
\Delta^{(k)}(0)=\sum_{i=1}^{5} a_{i} \sum_{n=0}^{k} C_{k}^{n}\left[\frac{\mathrm{d}^{n}\left[\Omega_{i}(z)\right]^{-1}}{\mathrm{~d} t^{n}}\right]_{t=0}\left[\frac{\mathrm{d}^{k-n} g_{i}(z)}{\mathrm{d} t^{k-n}}\right]_{t=0},
$$

and finally the conditions (45) can be written as

$$
\sum_{i=1}^{5} \sum_{n=0}^{k} b_{k n}^{(i)} c_{n, i}=s_{k}, \quad k=1, . ., K
$$

where the numbers $b_{k n}^{(i)}$ can be obtained in a straightforward way using Eq. (48), the definition (41) of the functions $\Omega_{i}(z)$ and the connection (23) between the variables $t$ and $z$. Of course, for each $k$ only the Taylor coefficients $c_{n, i}$ with $n \leq k$ contribute to $\Delta^{(k)}(0)$.

Our objective is to test the consistency of the relations (45) with the inequality (24) provided by the dispersive formalism. The problem can be easily solved, since we expressed both these conditions in terms of the Taylor coefficients $c_{n, i}$, transforming in this way a functional problem into an algebraic one. We notice that the first coefficients

$$
c_{0, i}=g_{i}(0)=\Omega_{i}(0) F_{i}(0), \quad i=1, . ., 5
$$

can be computed using the relations (4) and (41). The remaining coefficients $c_{n, i}, n \geq 1$ are free. The relations (49) can be viewed as a set of $N$ linear constraints for these coefficients, which satisfy in addition the quadratic condition (44). A simple way to test the consistency of these relations for a given set of numbers $s_{k}, k=1, \ldots, K$ is to define the quantity

$$
\mu_{0}^{2}=\min _{c_{n, i}} \sum_{i=1}^{5} \sum_{n=0}^{\infty} c_{n, i}^{2}
$$


where the minimization is with respect to the coefficients $c_{n, i}, n \geq 1, i=1, \ldots .5$ which satisfy the set of linear conditions (49). It is clear that, if the minimal value $\mu_{0}^{2}$ is greater than one, the conditions (44) and (49) can not be satisfied simultaneously, while if $\mu_{0}^{2}$ is less than one the conditions are satisfied by at least one set of coefficients $c_{n, i}$. A more detailed reasoning along these lines [34] shows that the inequality

$$
\mu_{0}^{2} \leq 1
$$

is a necessary and sufficient condition for the consistency of the relations (44) and (49).

In order to solve the minimization problem (51) with the constraints (49) we apply the technique of Lagrange multipliers. The Lagrangean of the problem is:

$$
\mathcal{L}=\sum_{i=1}^{5} c_{0, i}^{2}+\sum_{i=1}^{5} \sum_{n=1}^{\infty} c_{n, i}^{2}-2 \sum_{k=1}^{K} \lambda_{k}\left[\sum_{i=1}^{5} b_{k 0}^{(i)} c_{0, i}+\sum_{i=1}^{5} \sum_{n=1}^{K} b_{k n}^{(i)} c_{n, i}-s_{k}\right]
$$

where $\lambda_{k}, k=1, . . K$ are Lagrange multipliers. In (53) we indicated separately the coefficients $c_{0, i}$ which are known. Moreover, for convenience we extended the sum over $n$ in the last term up to $n=K$, setting $b_{k n}^{(i)}=0$ for $n>k$. The Lagrangean $\mathcal{L}$ is a convex function of the coefficients $c_{n, i}$, its minimal value being given by the equations

$$
\frac{\partial \mathcal{L}}{\partial c_{n, i}}=0, \quad n=1, \ldots \infty ; i=1, . .5
$$

The solution of these equations is

$$
\begin{aligned}
c_{n, i} & =\sum_{k-1}^{K} \lambda_{k} b_{k n}^{(i)}, \quad n \leq K, \quad i=1, . .5, \\
c_{n, i} & =0, \quad n \geq K+1, \quad i=1, . .5 .
\end{aligned}
$$

By introducing this solution in the conditions (45) we obtain the following system of equations for the Lagrange multipliers $\lambda_{k}$ :

$$
\sum_{m=1}^{K} U_{k m} \lambda_{m}=\tilde{s}_{k}, \quad k=1, \ldots K
$$

where the matrix $U$ is defined as

$$
U_{k m}=\sum_{i=1}^{5} \sum_{j=1}^{K} b_{k j}^{(i)} b_{m j}^{(i)}, \quad k, m=1, \ldots K
$$

and the numbers $\tilde{s}_{k}$ are related to the derivatives of the Sirlin function $s_{k}$ defined in (45) by

$$
\tilde{s}_{k}=s_{k}-\sum_{i=1}^{5} b_{k 0}^{(i)} c_{0, i}, \quad k=1, \ldots K
$$


The system (56) has the solution

$$
\lambda_{k}=\sum_{m=1}^{K}\left(U^{-1}\right)_{k m} \tilde{s}_{m}, \quad k=1, \ldots K,
$$

where $U^{-1}$ is the inverse of the matrix $U$. By introducing these expressions in (55) we obtain the optimal coefficients $c_{n, i}$ for $1 \leq n \leq K$ as

$$
c_{n, i}=\sum_{m=1}^{K} \tilde{s}_{m} \sum_{k=1}^{K}\left(U^{-1}\right)_{k m} b_{k n}^{(i)}, \quad n=1, \ldots K ; i=1, \ldots 5 .
$$

With these coefficients for $n \leq K$ and $c_{n, i}=0$ for $n \geq K+1$ according to (55) we obtain after a straightforward calculation the following compact form the solution $\mu_{0}^{2}$ of the minimization problem (51):

$$
\mu_{0}^{2}=\sum_{i=1}^{5} c_{0, i}^{2}+\sum_{j, k=1}^{K}\left(U^{-1}\right)_{j k} \tilde{s}_{j} \tilde{s}_{k}
$$

and the consistency condition (52) becomes

$$
\sum_{i=1}^{5} c_{0, i}^{2}+\sum_{j, k=1}^{K}\left(U^{-1}\right)_{j k} \tilde{s}_{j} \tilde{s}_{k} \leq 1
$$

We recall that in this inequality the coefficients $c_{0, i}$ are known according to (50) from the normalization of the form factors at $t=0$, and the matrix $U$ can be explicitely computed using (57) and the numbers $b_{k n}^{(i)}$ appearing in (49). We mention that these numbers include the physical input on unitarity and QCD , contained in the outer functions $w_{i}(z)$, and the phase of the kaon form factors along the unphysical region entering the outer functions $O_{i}(t)$ and $\omega_{i}(t)$. As for the quantities $\tilde{s}_{k}$, they are related to the derivatives $s_{k}$ of the Sirlin function, according to (58). With this input the inequality (62) is a constraint for the derivatives $s_{k}$ of the Sirlin function $\Delta(t)$ at $t=0$, defined in (45).

For illustration we evaluated the quantity $\mu_{0}^{2}$ for $K=3$, using the value $s_{1}=0.0033 \mathrm{fm}^{2}$ obtained recently in [6] and several choices for the higher derivatives $s_{2}$ and $s_{3}$. A few results are listed in Table 1 . The sets of values for which $\mu_{0}^{2}$ is less than 1 are consistent with the dispersive bounds (in particular, this is true for $s_{2}=s_{3}=0$ and small deviations from these values, showing that Sirlin's theorem is verified within the present formalism). We give also some pairs $\left(s_{2}, s_{3}\right)$ for which the quantity $\mu_{0}^{2}$ is greater than one, and therefore the inequality (62) is violated. The results show an adequate consistency between the requirements of Sirlin's theorem on one hand, and QCD and analyticity on the other. However, in order to exploit the dispersive inequalities we had to adopt a model for the phase of the kaon form factors along the unphysical region. The presence of the unphysical cut reduces therefore the model independence of the conclusions. 


\begin{tabular}{|c|c|c|}
\hline$s_{2}\left(\mathrm{fm}^{4}\right)$ & $s_{3}\left(\mathrm{fm}^{6}\right)$ & $\mu_{0}^{2}$ \\
\hline 0.0 & 0.0 & 0.04 \\
\hline 0.1 & -0.1 & 0.69 \\
\hline 0.1 & 0.1 & 0.06 \\
\hline-0.1 & 0.1 & 0.97 \\
\hline-0.01 & 0.3 & 1.07 \\
\hline 0.05 & -0.3 & 1.26 \\
\hline-0.1 & 0.5 & 4.71 \\
\hline
\end{tabular}

Table 1: The quantity $\mu_{0}^{2}$ defined in (61) for $s_{1}=0.0033 \mathrm{fm}^{2}[6]$ and several values of the derivatives $s_{2}$ and $s_{3}$ of the Sirlin function. The values consistent with QCD and analyticity correspond to $\mu_{0}^{2} \leq 1$.

\section{Bounds on the Taylor coefficients of the pion form factor at zero momentum}

In this Section we shall apply the dispersive formalism to the pion electromagnetic form factor $F_{\pi}(t)$. We consider the Taylor expansion of this function around the origin $t=0$ $[1]$

$$
F_{\pi}(t)=1+\frac{1}{6} r_{\pi}^{2} t+c t^{2}+d t^{3}+\ldots \ldots,
$$

where $r_{\pi}^{2}$ is the radius of the charge distribution. In CHPT the calculation of the Taylor coefficients requires the evaluation of higher pion loops, which introduce arbitrary renormalization constants [2], [5]. One-loop CHPT [1] predicts $c \approx 0.626 \mathrm{GeV}^{-4}, d \approx 2.30 \mathrm{GeV}^{-6}$. At two - loop level the coefficient $c$ can not be calculated as it depends on an arbitrary renormalization constant, and $d=4.1 \mathrm{GeV}^{-6}[5]$. A fit of the ALEPH data [35] on the hadronic $\tau$ decay rate with a Gounaris-Sakurai formula [36] (equivalent to the Padé version of the one-loop CHPT) gives $c=3.72 \mathrm{GeV}^{-4}, d=9.80 \mathrm{GeV}^{-6}$. Similar values $c=3.9 \mathrm{GeV}^{-4}, d=9.70 \mathrm{GeV}^{-6}$ were obtained in [37] by usual dispersion relations. Other values proposed in the literature are: $c=4.1 \mathrm{GeV}^{-4}$ [39], and $c=-7.5 \mathrm{GeV}^{-4}, d=62.5 \mathrm{GeV}^{-6}[38]$.

In what follows we shall prove that the dispersive formalism imposes nontrivial constraints on the Taylor expansion (63), especially when combined with additional information about the form factor along a part of the time like region. For the present purpose we keep only the contribution of the pion form factor in the unitarity inequality (11), neglecting the positive terms due to the $K$ mesons. In this case the inequality (24) becomes

$$
\frac{1}{2 \pi} \int_{0}^{2 \pi}\left|w_{\pi}(\zeta) F_{\pi}(\zeta)\right|^{2} \mathrm{~d} \theta \leq 1, \quad \zeta=\exp (i \theta)
$$


where the outer function $w_{\pi}(z)$ is

$$
w_{\pi}(z)=\frac{\left(1-d_{\pi}\right)^{2}}{16} \sqrt{\frac{1}{6 \pi t_{\pi} \Pi_{\text {elm }}^{\prime}\left(-Q^{2}\right)}} \frac{(1+z)^{2} \sqrt{1-z}}{\left(1-z d_{\pi}\right)^{2}},
$$

with $d_{\pi}=d_{1}$ defined in (28). If we use as an alternative input the lower bound on the hadronic contribution to the muon anomaly, the function $w_{\pi}(z)$ is

$$
w_{\pi}(z)=\frac{1}{16} \sqrt{\frac{1}{6 \pi t_{\pi} a_{\mu}^{(h)}}}(1+z)^{2} \sqrt{1-z} w_{\mathcal{K}}(z),
$$

where $w_{\mathcal{K}}(z)$ was defined below $(30)$. We consider now the function

$$
g(z)=w_{\pi}(z) F_{\pi}(z),
$$

which is real analytic in the unit disk $|z|<1$ and can be expanded as

$$
g(z)=\sum_{n=0}^{\infty} c_{n} z^{n}
$$

with real coefficients $c_{n}=c_{n}^{*}$. Then the condition (64) becomes

$$
\sum_{n=0}^{\infty} c_{n}^{2} \leq 1
$$

A constraint on the first $N$ coefficients appearing in the Taylor expansion (63) is immediately obtained by noticing that (69) implies

$$
\sum_{n=0}^{N} c_{n}^{2} \leq 1
$$

The first $N$ coefficients $c_{n}$ can be expressed in a straightforward way in terms of the first $N$ Taylor coefficients appearing in (63) . The explicit relations are obtained easily using the definition (67) and the conformal mapping (23). For instance, $c_{0}=w_{\pi}(0)$, $c_{1}=w_{\pi}^{\prime}(0)-2 r_{\pi}^{2} t_{\pi} w_{\pi}(0) / 3$ etc, where the function $w_{\pi}(z)$ is defined in (65) and the derivatives are with respect to $z$. If we use as input the lower bound on the muon anomaly, the function $w_{\pi}(z)$ has the expression (66).

In particular, for $N=3$ and a fixed value of the charge radius $r_{\pi}^{2}$ the inequality (70) defines an allowed domain in the plane of the coefficients $c$ and $d$ appearing in the Taylor expansion (63). In Fig.1, the interior of the larger ellipse is the domain obtained using the standard value $r_{\pi}^{2}=0.42 \mathrm{fm}^{2}$ and the QCD condition (15). This domain is very large, but one can see a certain correlation among the values of the Taylor coefficients. This feature becomes more stringent when higher derivatives of the form factor are taken into account. For comparison we indicated also in Fig. 1 the allowed domain obtained from the condition (20) on the muon anomaly. This domain is smaller, and in particular it excludes a pair of values for $c$ and $d$ proposed recently in [38]. In the next subsections we shall improve the above bounds by implementing informations about the phase and the modulus of the pion form factor along a part of the unitarity cut. 


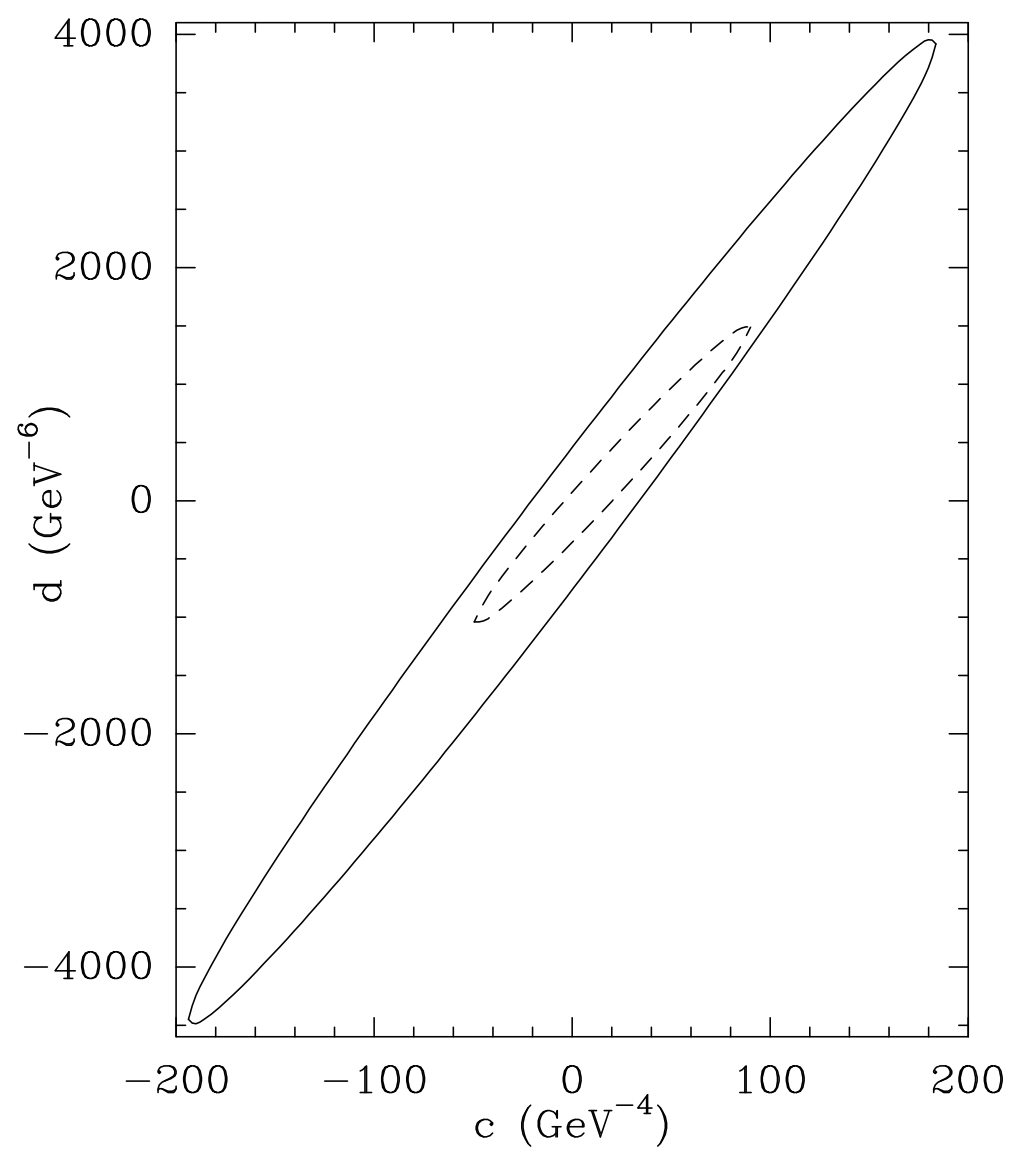

Figure 1: Allowed domains for the Taylor coefficients $c$ and $d$ of the pion electromagnetic form factor obtained from (70). The large ellipse is obtained using as input the QCD expansion of the polarization function, the small one using the lower bound on the muon hadronic anomaly. 


\subsection{Improved bounds using the phase of the form factor along a part of the cut}

According to Watson theorem [40], along the elastic region $t_{\pi}<t<16 m_{\pi}^{2}$ the phase of the pion electromagnetic form factor coincides with the phase $\delta_{1}^{1}(t)$ of the $L=1, I=1$ partial wave amplitude of the $\pi \pi$ scattering. This amplitude was calculated in the frame of CHPT up to two loops [5]. The expansion upon chiral loops is assumed to describe correctly the phase up to $\approx 0.400 \mathrm{GeV}$. On the other hand, precise experimental data are available above $\approx 0.600 \mathrm{GeV}$ [41]. New planned experiments on $K_{l 4}$ decay [42], [43], [44] will provide accurate information about the low energy $\pi \pi$ scattering amplitudes.

It is of interest to incorporate in the dispersive formalism for the pion form factor the additional information on the phase. Unlike the situation encountered in Section 3, where the knowledge of the phase allowed us to remove the unphysical cut below the unitarity threshold, in the present case we must implement the phase along a part of the unitarity cut. We treat the problem adapting mathematical techniques used in [45]-[48]. We assume that

$$
\operatorname{Arg}\left[F_{\pi}(t+i \epsilon)\right]=\delta_{1}^{1}(t), \quad t_{\pi} \leq t \leq t_{i n}
$$

where $\delta_{1}^{1}(t)$ is a known function and $t_{\text {in }}$ denotes the threshold of the inelastic cut or a certain point up to which the phase is supposed to be known. As we shall discuss below, the technique can be easily adapted to more general situations, for instance when the phase is given along the range $t_{1} \leq t \leq t_{\text {in }}$ with $t_{1}>t_{\pi}$, or along a region consisting of two disjoint parts, $t_{\pi} \leq t \leq t_{1}$ and $t_{2} \leq t \leq t_{i n}$, with $t_{2}>t_{1}$.

We first express the condition (71) in the variable $z$ defined in (23). To this end we recall that the branch point $t_{\pi}$ is mapped onto the point $z=-1$, and the upper edge of the unitarity cut in the $t$ plane becomes the lower unit semicircle in the $z$ plane. We denote by $z_{i n}=\exp \left(i \theta_{i n}\right)$ the image of the point $t_{i n}-i \epsilon$ in the $z$-plane, and let $\Gamma=\left\{\theta: \theta_{\text {in }}<\theta<2 \pi-\theta_{\text {in }}\right\}$. Then the condition (71) becomes

$$
\begin{aligned}
& \lim _{r \rightarrow 1} \operatorname{Arg}\left[F_{\pi}\left(r \mathrm{e}^{i \theta}\right)\right]=-\delta_{1}^{1}(\theta), \quad \theta_{i n} \leq \theta \leq \pi, \\
& \lim _{r \rightarrow 1} \operatorname{Arg}\left[F_{\pi}\left(r \mathrm{e}^{i \theta}\right)\right]=\delta_{1}^{1}(\theta), \quad \pi \leq \theta \leq 2 \pi-\theta_{i n},
\end{aligned}
$$

where we denoted $\delta_{1}^{1}(\theta)=\delta_{1}^{1}(t(\theta))$, with $t(\theta)=t_{\pi}+t_{\pi} \operatorname{cotg}^{2}(\theta / 2)$ as follows from (23). We took into account the fact that the form factor is an analytic function of real type, which means that its phase is an odd function of $\theta$, i.e. it satisfies the relation $\delta_{1}^{1}(\theta)=$ $-\delta_{1}^{1}(2 \pi-\theta)$. It is useful to note that the same property holds also for the imaginary part of the form factor, while the real part and the modulus are even functions of $\theta$.

In what follows we shall derive the allowed domain for the Taylor coefficients of the expansion (63), taking into account the inequality (64) provided by the dispersive formalism, and the additional relations (72). As usual, the information about the phase is implemented by means of an Omnès function [33]. We can use the definition given in 
(35), or equivalently we define

$$
\mathcal{O}_{\pi}(z)=\exp \left[\frac{i}{\pi} \int_{0}^{2 \pi} \mathrm{d} \theta \frac{\bar{\delta}_{1}^{1}(\theta)}{1-z \mathrm{e}^{-i \theta}}\right]
$$

where $\bar{\delta}_{1}^{1}(\theta)$ is a Lipschitz continuous function such that $\bar{\delta}_{1}^{1}(\theta)=-\delta_{1}^{1}(\theta)$ for $\theta_{\text {in }}<\theta<$ $\pi, \bar{\delta}_{1}^{1}(\theta)=\delta_{1}^{1}(\theta)$ for $\pi<\theta<\pi+\theta_{\text {in }}, \bar{\delta}_{1}^{1}(\theta)$ being arbitrary outside $\Gamma$. We shall see below that the results are independent on the choice of $\bar{\delta}_{1}^{1}(\theta)$ outside $\Gamma$. Using the PlemeljPrivalov relation [49]

$$
\lim _{r \rightarrow 1} \frac{1}{\pi} \int_{0}^{2 \pi} \mathrm{d} \theta^{\prime} \frac{F\left(\theta^{\prime}\right)}{1-r \mathrm{e}^{i\left(\theta-\theta^{\prime}\right)}}=F(\theta)+\frac{1}{\pi} \int_{0}^{2 \pi} \mathrm{d} \theta^{\prime} \frac{F\left(\theta^{\prime}\right)}{1-\mathrm{e}^{i\left(\theta-\theta^{\prime}\right)}},
$$

where the last integral denotes the Principal Value, one can show that along the interval $\Gamma$ the phase of the function $\mathcal{O}_{\pi}$ coincides with the phase of the form factor. Therefore, by multiplying $F_{\pi}(z)$ with $\left[O_{\pi}(z)\right]^{-1}$ the phases compensate each other and the product is real along the elastic part of the cut. In the variable $z$ this condition has the form

$$
\operatorname{Im} \lim _{r \rightarrow 1}\left[\frac{1}{\mathcal{O}_{\pi}\left(r \mathrm{e}^{i \theta}\right)} F_{\pi}\left(r \mathrm{e}^{i \theta}\right)\right]=0, \quad \theta \in \Gamma .
$$

By recalling the definition (67) of the function $g(z)$ and its power expansion (68), we write the condition (75) in the form

$$
\begin{array}{r}
\operatorname{Im} \lim _{r \rightarrow 1}\left[\frac{1}{w_{\pi}\left(r \mathrm{e}^{i \theta}\right) \mathcal{O}_{\pi}\left(r \mathrm{e}^{i \theta}\right)} g\left(r \mathrm{e}^{i \theta}\right)\right]= \\
\sum_{n=0}^{\infty} c_{n} \operatorname{Im} \lim _{r \rightarrow 1}\left[\left[W\left(r \mathrm{e}^{i \theta}\right)\right]^{-1} r^{n} \mathrm{e}^{i n \theta}\right]=0, \quad \theta \in \Gamma,
\end{array}
$$

where $W(\zeta)$ is defined as

$$
W(\zeta)=w_{\pi}(\zeta) \mathcal{O}_{\pi}(\zeta), \quad \zeta=\mathrm{e}^{i \theta}
$$

The allowed domain of the Taylor coefficients of the pion form factor, which satisfy the conditions (64) and (75) can be found, like in Section 3, by means of an optimization problem. We first recall that the conditions (64) and (75) were written in the equivalent forms (69) and (76) respectively, in terms of the Taylor coefficients $c_{n}$. We consider then the quantity

$$
\mu_{0}^{2}=\min _{c_{n}} \sum_{n=0}^{\infty} c_{n}^{2}
$$

where the minimization is with respect to the coefficients $c_{n}$ which satisfy the condition (76). As in Section 3 one can show that the inequality (52) is a necessary and sufficient condition for the consistency of the relations (69) and (76). 
We solve the constrained minimization problem (78) with the generalized Lagrange theory of optimization, based on Hahn-Banach theorem [50]. The Lagrangean is

$$
\mathcal{L}=\sum_{n=0}^{\infty} c_{n}^{2}+\frac{2}{\pi} \sum_{n=0}^{\infty} c_{n} \lim _{r \rightarrow 1} \int_{\Gamma} \lambda(\theta)|W(\theta)| \operatorname{Im}\left[[W(\theta)]^{-1} r^{n} \mathrm{e}^{i n \theta}\right] \mathrm{d} \theta,
$$

where the function $\lambda(\theta)$ is a generalized Lagrange multiplyer [50]. We can assume without losing generality that it is an odd function, since its product with another odd function (the imaginary part of an analytic function of real type) is integrated along a symmetric interval $\Gamma$. We denoted for simplicity $W(\theta)=W(\exp (i \theta))$. The numerical factor in front of the integral in (79) and the modulus $|W(\theta)|$ inside the integral were introduced explicitely for convenience.

We assume now that the first $N$ coefficients $c_{n}$ have prescribed values, and perform the minimization of the Lagrangean with respect to the remaining coefficients $c_{n}, n \geq N+1$. The minimum condition

$$
\frac{\partial \mathcal{L}}{\partial c_{n}}=0, \quad n \geq N+1
$$

gives the optimal coefficients

$$
c_{n}=-\frac{1}{\pi} \lim _{r \rightarrow 1} \int_{\Gamma} \lambda(\theta)|W(\theta)| \operatorname{Im}\left[[W(\theta)]^{-1} r^{n} \mathrm{e}^{i n \theta}\right] \mathrm{d} \theta, \quad n \geq N+1 .
$$

This expression can be written equivalently as

$$
c_{n}=-\frac{i}{\pi} \lim _{r \rightarrow 1} \int_{\Gamma} \lambda(\theta)|W(\theta)|\left[W^{*}(\theta)\right]^{-1} r^{n} \mathrm{e}^{-i n \theta} \mathrm{d} \theta, \quad n \geq N+1,
$$

where we added a term which has a vanishing contribution by parity arguments.

In order to find the Lagrange multiplier $\lambda(\theta)$ we introduce the optimal coefficients $c_{n}$ in the constraint (76). Recalling that the first $N$ coefficients $c_{n}$ are assumed to have prescribed values, we write the condition (76) in the form

$$
\sum_{n=0}^{N} c_{n} \operatorname{Im}\left[\frac{\mathrm{e}^{i n \theta}}{W(\theta)}\right]-\operatorname{Im} \lim _{r \rightarrow 1}\left[\frac{i}{\pi} \frac{1}{W(\theta)} \int_{\Gamma} \lambda\left(\theta^{\prime}\right) \frac{\left|W\left(\theta^{\prime}\right)\right|}{W^{*}\left(\theta^{\prime}\right)} \frac{\mathrm{e}^{i(N+1)\left(\theta-\theta^{\prime}\right)}}{1-r \mathrm{e}^{i\left(\theta-\theta^{\prime}\right)}} \mathrm{d} \theta^{\prime}\right]=0, \theta \in \Gamma .
$$

It is useful to write

$$
W(\theta)=|W(\theta)| \mathrm{e}^{i \Phi(\theta)},
$$

where from (77) it follows that

$$
\Phi(\theta)=\phi(\theta)+\bar{\delta}_{1}^{1}(\theta)
$$

Here $\phi(\theta)$ is the phase of the outer function $w_{\pi}(z)$, and $\bar{\delta}_{1}^{1}(\theta)$ is connected to the phase of the pion form factor as explained below (73). By inserting (84) in the relation (83) and 
applying the Plemelj-Privalov relation (74) we obtain after a straightforward calculation the equation

$$
\begin{aligned}
& \sum_{n=0}^{N} c_{n} \sin [n \theta-\Phi(\theta)]-\lambda(\theta) \\
& +\frac{1}{2 \pi} \int_{\Gamma} \lambda\left(\theta^{\prime}\right) \frac{\sin \left[\left(N+\frac{1}{2}\right)\left(\theta-\theta^{\prime}\right)-\Phi(\theta)+\Phi\left(\theta^{\prime}\right)\right]}{\sin \left[\frac{\theta-\theta^{\prime}}{2}\right]} \mathrm{d} \theta^{\prime}=0, \theta \in \Gamma,
\end{aligned}
$$

where the last integral is defined as the Principal Value. We obtained therefore a singular integral equation for the Lagrange multiplier $\lambda(\theta)$. If the phase $\Phi(\theta)$ is Lipschitz continuous, the equation is of Fredholm type and can be solved by standard techniques. After solving this equation, the minimal norm $\mu_{0}^{2}$ can be computed by inserting in (78) the prescribed values $c_{n}$ for $n \leq N$ and the optimal values (82) of $c_{n}$ for $n \geq N+1$. Taking into account the fact that $c_{n}=c_{n}^{*}$ we obtain

$$
\mu_{0}^{2}=\sum_{n=0}^{N} c_{n}^{2}+\lim _{r \rightarrow 1} \frac{1}{\pi^{2}} \lim _{r \rightarrow 1} \int_{\Gamma} \mathrm{d} \theta \int_{\Gamma} \mathrm{d} \theta^{\prime} \lambda(\theta) \lambda\left(\theta^{\prime}\right) \frac{|W(\theta)|}{W^{*}(\theta)} \frac{\left|W\left(\theta^{\prime}\right)\right|}{W^{*}\left(\theta^{\prime}\right)} \frac{\mathrm{e}^{i(N+1)\left(\theta-\theta^{\prime}\right)}}{1-r \mathrm{e}^{i\left(\theta-\theta^{\prime}\right)}} .
$$

By applying the Plemelj-Privalov relation (74) and using the integral equation (86) satisfied by the function $\lambda(\theta)$, we arrive finally at the expression

$$
\mu_{0}^{2}=\sum_{n=0}^{N} c_{n}^{2}+\sum_{n=0}^{N} \frac{c_{n}}{\pi} \int_{\Gamma} \lambda(\theta) \sin [n \theta-\Phi(\theta)] \mathrm{d} \theta
$$

Using the fact that $\lambda(\theta)$ and $\Phi(\theta)$ are odd functions, the integral equation (86) can be written in the form

$$
\begin{aligned}
& \sum_{n=0}^{N} c_{n} \sin [n \theta-\Phi(\theta)]-\lambda(\theta) \\
& +\frac{1}{2 \pi} \int_{\theta_{i n}}^{\pi} \lambda\left(\theta^{\prime}\right)\left[\frac{\sin \left[\left(N+\frac{1}{2}\right)\left(\theta-\theta^{\prime}\right)-\Phi(\theta)+\Phi\left(\theta^{\prime}\right)\right]}{\sin \left[\frac{\theta-\theta^{\prime}}{2}\right]}\right. \\
& \left.-\frac{\sin \left[\left(N+\frac{1}{2}\right)\left(\theta+\theta^{\prime}-2 \pi\right)-\Phi(\theta)-\Phi\left(\theta^{\prime}\right)\right]}{\sin \left[\frac{\theta+\theta^{\prime}}{2}-\pi\right]}\right] \mathrm{d} \theta^{\prime}=0, \quad \theta_{i n} \leq \theta \leq \pi,
\end{aligned}
$$

where

$$
\Phi(\theta)=\phi(\theta)-\delta_{1}^{1}(\theta), \quad \theta_{\text {in }} \leq \theta \leq \pi .
$$

Moreover, the expression (88) of $\mu_{0}^{2}$ can be written as

$$
\mu_{0}^{2}=\sum_{n=0}^{N} c_{n}^{2}+\sum_{n=0}^{N} \frac{2 c_{n}}{\pi} \int_{\theta_{i n}}^{\pi} \lambda(\theta) \sin [n \theta-\Phi(\theta)] \mathrm{d} \theta .
$$


We notice that the first term in (91) represents the unconstrained minimum, obtained from (70) if the coefficients $c_{n}$ for $n \geq N+1$ were free. The second term in the expression of $\mu_{0}^{2}$ is positive and represents the improvement brought by the knowledge of the phase of the form factor along the region $t_{\pi}<t<t_{i n}$.

It is important to emphasize that only the values of $\Phi(\theta)$ along the interval $\Gamma$ are required in the integral equation. Also, the modulus of the outer function does not appear (it was absorbed in the definition of the Lagrange multiplier $\lambda(\theta)$ ), which means that the results are independent of the choice of the function $\bar{\delta}_{1}^{1}$ outside the interval $\Gamma$.

The equations (89) - (91) provide a simple numerical procedure for finding the allowed domain of the coefficients of the Taylor expansion (63): one starts with a set of values for the first $N$ Taylor coefficients. Using the relations (67) and (68) one finds the corresponding coefficients $c_{n}$, which enter the integral equation (89). The solution $\lambda(\theta)$ of this equation is then used in (91) to evaluate the quantity $\mu_{0}^{2}$. Recalling that the allowed domain of the Taylor coefficients is described by the inequality (52), the values taken as input are accepted or rejected if $\mu_{0}^{2}$ is less or greater than unity, respectively.

From the above derivation the generalization to the case where the region $\Gamma$ consists of two disjoint subintervals $\Gamma_{1}$ and $\Gamma_{2}$ is straightforward. This case is of interest when using the phase given by CHPT along $\Gamma_{1}=\left\{t: t_{\pi}<t<(0.400 \mathrm{GeV})^{2}\right\}$ and the experimental data on $\Gamma_{2}=\left\{t:(0.600 \mathrm{GeV})^{2}<t<t_{i n}\right\}$. The resulting equations have the same form, the Lagrange multiplier being defined on $\Gamma_{1} \cup \Gamma_{2}$.

To illustrate how the bounds on the Taylor coefficients $c$ and $d$ are improved by the knowledge of the phase, we took the expression [30]

$$
\delta_{1}^{1}(t)=\operatorname{arctg}\left[\frac{m_{\rho} \Gamma_{\rho}(t)}{m_{\rho}^{2}-t}\right]
$$

where $\Gamma_{\rho}(t)$ was defined in (34). At low energies above the threshold, this phase coincides with the one loop CHPT expression [1]

$$
\delta_{1}^{1}(t)=\frac{t}{96 \pi f_{\pi}^{2}}\left(1-\frac{4 m_{\pi}^{2}}{t}\right)^{3 / 2}
$$

while for $t \geq(0.500 \mathrm{GeV})^{2}$ it is in very good agreement with the experimental data [41]. We assumed that the phase of the pion form factor coincides with (92) along the region $t_{\pi}<t<t_{i n}$, with $t_{i n}=0.8 \mathrm{GeV}^{2}$, which corresponds to $\theta_{i n}=0.6321$. As input for the bounds we use the OPE expansion of the polarization amplitude, which led to the domain represented by the interior of the large ellipse in Fig.1. In this case the outer function $w_{\pi}(z)$ is given in (65) and the function $\Phi(\theta)$ defined in (90) has the expression

$$
\Phi(\theta)=\frac{5 \theta-\pi}{4}+\operatorname{arctg}\left[\frac{2 d_{1} \sin \theta-d_{1}^{2} \sin 2 \theta}{1-d_{1} \cos \theta+d_{1}^{2} \cos 2 \theta}\right]-\delta_{1}^{1}(\theta), \quad \theta_{\text {in }} \leq \theta \leq \pi,
$$

with $d_{1}$ defined in (28). In Fig. 2 the interior of the ellipse is the allowed domain in the plane $(c, d)$, for $r_{\pi}^{2}=0.42 \mathrm{fm}^{2}$. Compared with the large ellipse in Fig.1 one can see 


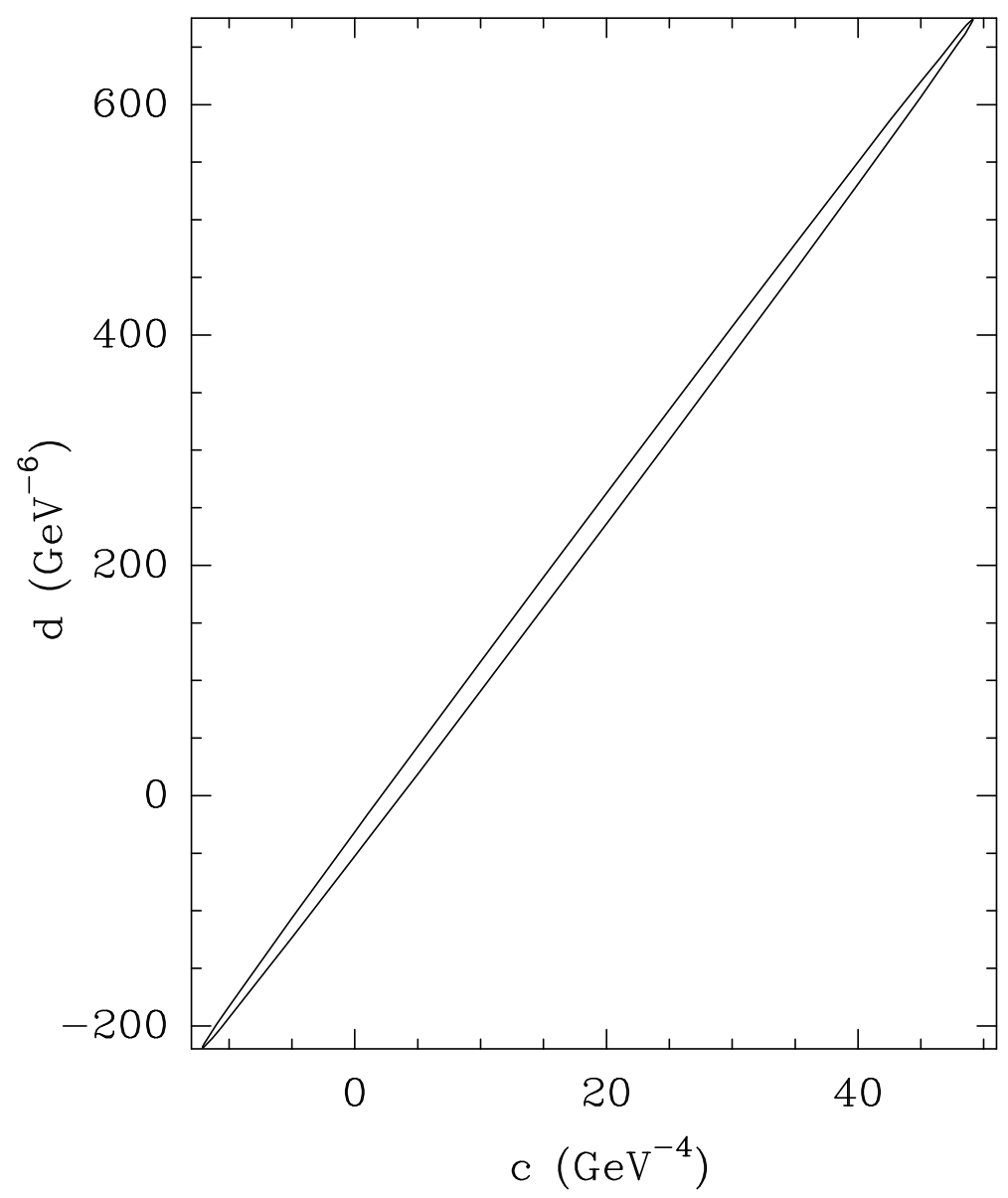

Figure 2: Improved domain for the Taylor coefficients $c$ and $d$ of the pion electromagnetic form factor, using the phase along a part of the boundary. 
the considerable improvement brought by the knowledge of the phase along a part of the unitarity cut. A more realistic calculation using CHPT along an interval $\Gamma_{1}$ and the experimental data on $\delta_{1}^{1}(t)$ along another interval $\Gamma_{2}$ is of interest and will provide precise model independent bounds on the Taylor coefficients.

\subsection{Improved bounds using information about the phase and the modulus along a part of the cut}

The modulus of the pion form factor along a part of the cut is known experimentally from the rate of $e^{+} e^{-}$annihilation into pions [51] and the hadronic $\tau$ decay [35]. High precision data are available especially in the range $0.3 \mathrm{GeV}^{2}<t<0.9 \mathrm{GeV}^{2}$. In this subsection we derive the allowed domain of the Taylor coefficients by including some information about the modulus of the pion form factor along a part of the cut.

It is a known mathematical fact that if the modulus and the phase of an analytic function are known exactly along a part of the boundary, then the function is in principle uniquely determined. Explicit formulas for recapturing an analytic function belonging to a certain class, from its restriction along a part of the boundary are available [52], [53] (see also [47] for explicit expressions and further references). These expressions are however very unstable numerically, reflecting the fact that the analytic continuation is an ill-posed problem [54]. More exactly, the formulas give the correct analytic continuation if the input values are known with infinite accuracy, but they lead to arbitrary predictions if these values are affected by errors.

In what follows we shall show that even a nonoptimal use of the input information about the phase and the modulus leads to a considerable improvement of the bounds on the Taylor coefficients. Unlike the case considered in the previous subsection, where the phase could be given along several disjoint intervals, the method described below requires the knowledge of the phase along the whole range $t_{\pi} \leq t \leq t_{i n}$. We start by writing the inequality (12) in the form

$$
\frac{1}{48 \pi^{2}} \int_{t_{i n}}^{\infty} \frac{\mathrm{d} t}{\left(t+Q^{2}\right)^{2}}\left(1-\frac{4 m_{\pi}^{2}}{t}\right)^{3 / 2}\left|F_{\pi}(t)\right|^{2} \leq \mathcal{M}\left(Q^{2}\right)
$$

where

$$
\mathcal{M}\left(Q^{2}\right)=\Pi_{\text {elm }}^{\prime}\left(-Q^{2}\right)-\frac{1}{48 \pi^{2}} \int_{t_{\pi}}^{t_{i n}} \frac{\mathrm{d} t}{\left(t+Q^{2}\right)^{2}}\left(1-\frac{4 m_{\pi}^{2}}{t}\right)^{3 / 2}\left|F_{\pi}(t)\right|^{2} .
$$

Assuming that the modulus $\left|F_{\pi}(t)\right|$ is known along $t_{\pi} \leq t \leq t_{\text {in }}$, the quantity $\mathcal{M}\left(Q^{2}\right)$ can be evaluated numerically. To be conservative, one can use a lower bound on the modulus, which does not spoil the inequality (95) and leads to a larger allowed domain for the Taylor coefficients.

In order to incorporate the information about the phase we use the Omnès function 
defined in (73), which can be written equivalently in the $t$ variable as

$$
O_{\pi}(t)=\exp \left[\frac{t}{\pi} \int_{t_{\pi}}^{\infty} \frac{\bar{\delta}_{1}^{1}\left(t^{\prime}\right)}{t^{\prime}\left(t^{\prime}-t\right)} \mathrm{d} t^{\prime}\right] .
$$

The properties of the function $\bar{\delta}_{1}^{1}(t)$ were discussed below (73), and we use it in order to remove the part of the cut below $t=t_{i n}$. More precisely, writing as in (36)

$$
F_{\pi}(t)=f_{\pi}(t) O_{\pi}(t)
$$

we see that the function $f_{\pi}(t)$ is real on the real axis below $t_{i n}$, since the phase of the form factor along $t_{\pi} \leq t \leq t_{i n}$ is compensated by the phase of $O_{\pi}$. Therefore $f_{\pi}(t)$ is analytic in the $t$-plane cut for $t>t_{i n}$, and satisfies on the cut the condition

$$
\frac{1}{48 \pi^{2}} \int_{t_{i n}}^{\infty} \frac{\mathrm{d} t}{\left(t+Q^{2}\right)^{2}}\left(1-\frac{4 m_{\pi}^{2}}{t}\right)^{3 / 2}\left|f_{\pi}(t)\right|^{2}\left|O_{\pi}(t)\right|^{2} \leq \mathcal{M}\left(Q^{2}\right),
$$

which follows from (95). But this condition can be simply exploited with the standard techniques described in subsection 2.3. We first map the $t$ plane cut for $t>t_{\text {in }}$ onto the unit disk in the $z$-plane, using the conformal mapping

$$
z(t)=\frac{\sqrt{t_{i n}-t}-\sqrt{t_{i n}}}{\sqrt{t_{i n}-t}+\sqrt{t_{i n}}} .
$$

We define as in (39) the outer function

$$
\omega_{\pi}(t)=\exp \left[\frac{\sqrt{t_{i n}-t}}{\pi} \int_{t_{i n}}^{\infty} \frac{\ln \left|O_{\pi}\left(t^{\prime}\right)\right|}{\sqrt{t^{\prime}-t_{i n}}\left(t^{\prime}-t\right)} \mathrm{d} t^{\prime}\right],
$$

such that $\left|\omega_{\pi}(t)\right|=\left|O_{\pi}(t)\right|$ for $t>t_{\text {in }}$, and the outer function similar to (65)

$$
\tilde{w}_{\pi}(z)=\frac{\left(1-\tilde{d}_{\pi}\right)^{2}}{16} \sqrt{\frac{1}{6 \pi t_{\pi} \mathcal{M}\left(Q^{2}\right)}} \frac{(1+z)^{2} \sqrt{1-z}}{\left(1-z \tilde{d}_{\pi}\right)^{2}},
$$

with

$$
\tilde{d}_{\pi}=\frac{\sqrt{t_{i n}+Q^{2}}-\sqrt{t_{i n}}}{\sqrt{t_{i n}+Q^{2}}+\sqrt{t_{i n}}} .
$$

By introducing a new function $\tilde{g}(z)$, defined as

$$
\tilde{g}(z)=\tilde{w}_{\pi}(z) \omega_{\pi}(z) f_{\pi}(z)=\tilde{w}_{\pi}(z) \omega_{\pi}(z)\left[O_{\pi}(z)\right]^{-1} F_{\pi}(z),
$$

the inequality (99) takes the canonical form

$$
\frac{1}{2 \pi} \int_{0}^{2 \pi}|\tilde{g}(\zeta)|^{2} \mathrm{~d} \theta \leq 1, \quad \zeta=\exp (i \theta)
$$




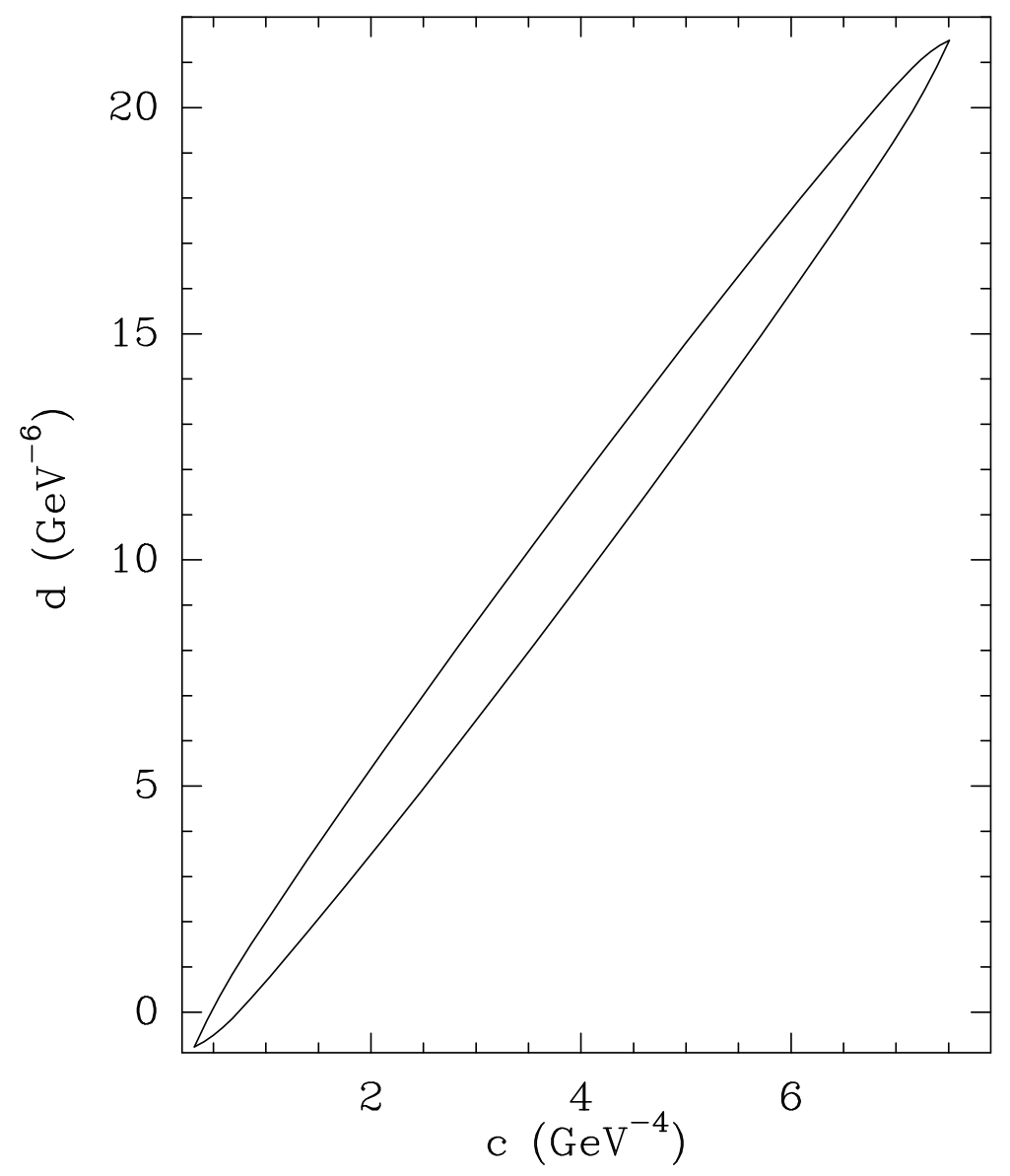

Figure 3: Improved domain for the Taylor coefficients $c$ and $d$ of the pion electromagnetic form factor, using the phase and the modulus along a part of the boundary.

By construction the function $\tilde{g}(z)$ is analytic in the disk $|z|<1$ and can be expanded as

$$
\tilde{g}(z)=\sum_{n=0}^{\infty} \tilde{c}_{n} z^{n}
$$

where the coefficients $\tilde{c}_{n}$ satisfy the inequality

$$
\sum_{n=0}^{\infty} \tilde{c}_{n}^{2} \leq 1
$$

which follows from (105). The first $N$ coefficients $\tilde{c}_{n}$ are connected in a straightfoward way through (104) to the first $N$ Taylor coefficients of the form factor $F_{\pi}(t)$ at $t=0$. Therefore, the inequality

$$
\sum_{n=0}^{N} \tilde{c}_{n}^{2} \leq 1
$$


which follows from (107), defines an allowed domain in the plane of these Taylor coefficients. In principle this domain can be further reduced: indeed, in addition to the boundary condition (99), the function $f_{\pi}$ defined in (98) has known error-affected values along the region $t_{\pi} \leq t \leq t_{i n}$, where it is holomorphic. The inclusion of this additional information is not trivial, and we will not treat this problem here, using the simple inequality (108). In particular, for $N=3$ and $r_{\pi}^{2}$ fixed, this inequality gives the allowed domain of the parameters $c$ and $d$ of the expansion (63).

In a numerical application we used the same phase $\delta_{1}^{1}(t)$ as in the preceeding subsection and the modulus $|F(t)|$ from the ALEPH data [35]. Using $Q^{2}=2 \mathrm{GeV}^{2}$ and $t_{i n}=$ $0.8 \mathrm{GeV}^{2}$, we obtain for the quantity $\mathcal{M}\left(Q^{2}\right)$ defined in (96) the estimate $\mathcal{M}\left(Q^{2}\right)=$ $0.00689 \mathrm{GeV}^{-2}$. The result is not sensitive to the experimental uncertainties above the threshold $t_{\pi}$, due to the phase space factor in the unitarity integral appearing in (96). Reducing the value of $|F(t)|$ along $t_{\pi} \leq t \leq t_{\text {in }}$ by $5 \%$ leads to an increase of $\mathcal{M}\left(Q^{2}\right)$ by about $3 \%$ and to bounds on the coefficients $c$ and $d$ weaker by about $2 \%$. The interior of the ellipse shown in Fig.3 indicates the allowed domain obtained in this conservative situation.

The combined information on the phase and the modulus is seen to restrict in an impressive way the values of the Taylor coefficients of the pion form factor. The allowed range of $c$ is $(0.25,7.57)$, and for each $c$ the range of the values of $d$ is very narrow. For instance, for $c=3.85$ the parameter $d$ is restricted to the interval $(9.02,11.30)$. The values $c=-7.5 \mathrm{GeV}^{-4}, d=62.5 \mathrm{GeV}^{-6}$ adopted in [38] are outside the allowed domain. We recall that these strong bounds are obtained with no specific parametrization and without any assumption about the high energy behaviour of the form factor.

\section{Conclusions}

In the present paper we investigated the form factors of the light pseudoscalar mesons in a dispersive formalism which uses as input the OPE expansion of the QCD polarization functions, combined with hadronic unitarity and analyticity. We derived constraints on the size and shape of the form factors, which are of interest in particular for testing the low energy predictions of CHPT. Generalizations of the original mathematical techniques, suitable for including additional informations about the form factors were developed.

In Section 3 we performed a test of Sirlin's theorem [7], [6], which requires the vanishing of a certain combination of form factors which is free of arbitrary renormalization constants in CHPT at $t \neq 0$. To this end we treated simultaneously in the dispersive formalism all the electroweak form factors of the $\pi$ and $K$ mesons. A difficulty is the large unphysical cut of the kaon electromagnetic form factors, which spoils the model independence of the results. This is in contrast with the case of heavy mesons, where the weak form factors of the ground state and of the excited ones have very close branch points and the unphysical cuts are well approximated by a few number of poles. In that case the simultaneous treatment of several form factors related among them by heavy quark symmetry led to strong model independent bounds near zero recoil [16], [20], [21]. In the present paper 
we adopted for the phase of the kaon form factors along the unphysical cut a model inspired from the resonance chiral effective theory, which turned out to be consistent with QCD and Sirlin's theorem. This subsection has however mainly a pedagogical character, showing how to combine in an optimal way the dispersive formalism with the Omnès functions and the technique of Lagrange multipliers for the constraints at interior points.

The main results are presented in Section 4, where we derived model independent constraints on the Taylor coefficients of the pion electromagnetic form factor. We showed how to implement in the dispersive formalism the knowledge of the phase of the form factor along a part of the unitarity cut, even when this part consists of several disjoint intervals. To this end we applied the Lagrange theory for functional optimization, which led to an integral equation for the generalized Lagrange multiplier. The knowledge of the phase considerably improves the simple bounds on the higher Taylor coefficients yielded by the dispersive formalism. In the present paper we illustrated this statement using a realistic model of the phase [30], which reproduces the one-loop CHPT expression [1] below $0.400 \mathrm{GeV}$ and the present experimental data [41] above $0.500 \mathrm{GeV}$. The accurate data provided on the phase $\delta_{1}^{1}$ by the future $K_{l 4}$ experiments [42],[43] will be a precious input to the formalism. In Section 4 we obtained also improved constraints on the Taylor coefficients if both the phase and the modulus of the form factor are given along a part of the cut, even if this information is used in an nonoptimal way. The interior of the ellipse shown in Fig. 3 represents the allowed domain of the coefficients $c$ and $d$ entering the Taylor expansion (63), obtained with the phase discussed above and the modulus from the hadronic $\tau$ decay rate [35] along $t_{\pi} \leq t \leq 0.8 \mathrm{GeV}^{2}$. Once this input information is adopted, the bounds are model independent, since they are not based on specific parametrizations and are free of any assumption about the high energy behaviour of the form factors. It is of interest to apply the techniques described in this paper to the higher Taylor coefficients. The results obtained so far suggest that strong correlations among these coefficients are expected, leading to restrictions on the arbitrary renormalization constants of CHPT.

Acknowledgements: I am pleased to thank Prof. H. Leutwyler for very useful discussions and the kind hospitality at the Institute of Theoretical Physics of the University of Berne, and Dr. C. Bourrely for many suggestions and a careful reading of the manuscript. The support of the Swiss National Science Foundation in the program CSR CEEC/ NIS, Contract No 7 IP 051219 is gratefully acknowledged.

\section{References}

[1] J. Gasser and H. Leutwyler, Ann. Phys. 158, 142 (1984); Nucl. Phys. B250 , 465 (1985).

[2] J. Gasser and U. Meissner, Nucl. Phys. B357, 90 (1991). 
[3] J. Bijnens, P. Colangelo, G. Ecker, J. Gasser and M. E. Sainio, Phys. Lett. B374, 210 (1996); Nucl. Phys. B508, 263 (1997); ibid. B517, 639 (1998) (E).

[4] G. Colangelo, M. Finkemeier and R. Urech, Phys. Rev. D54, 4403 (1996).

[5] J. Bijnens, P. Colangelo and P. Talavera, JHEP 9805:014 (1998).

[6] P. Post, K. Schilcher, Phys. Rev. Lett. 79, 4088 (1997).

[7] A. Sirlin, Ann. Phys. 61, 294 (1970); Phys. Rev. Lett. 43, 904 (1979).

[8] M. Ademollo, R. Gatto, Phys. Lett. 13, 264 (1964).

[9] T. N. Truong, Phys. Rev. Lett. 67, 2260 (1991).

[10] T. Hannah, Phys. Rev. D55, 5613 (1997).

[11] N.N. Meiman, Sov. Phys. JETP 17, 830 (1963).

[12] S. Okubo, Phys. Rev. D 3, 2807 (1971);

S. Okubo and I. Shih, Phys. Rev. D 4, 2020 (1971).

[13] C. Bourrely, B. Machet and E. de Rafael, Nucl. Phys. B189, 157 (1981).

[14] E.de Rafael and J.Taron, Phys. Lett. B282, 215 (1992);

[15] E.de Rafael and J.Taron, Phys. Rev. D50, 373 (1994).

[16] I.Caprini, Z.Phys. C61, 651 (1994).

[17] I. Caprini and C. Macesanu, Phys. Rev. D54, 5686 (1996).

[18] C.G.Boyd, B.Grinstein and R.F.Lebed, Phys. Lett. B353, 306 (1995).

[19] I. Caprini and M. Neubert, Phys. Lett. B380, 376 (1996).

[20] C.G.Boyd, B.Grinstein and R.F.Lebed, Phys. Rev. D56, 6895 (1997).

[21] I. Caprini, L.Lellouch and M. Neubert, Nucl. Phys. B530, 153 (1998).

[22] N. Isgur and M.B. Wise, Phys. Lett. B232, 113 (1989); B237, 527 (1990).

[23] M. Neubert, Phys. Rep. 245, 259 (1994).

[24] W.W. Buck and R. F. Lebed, Phys. Rev. D58, 056001 (1998).

[25] E.Braaten, S. Narison and A. Pich, Nucl. Phys. B373, 581 (1992).

[26] M. A. Shifman, A.I. Vainshtein and V. I. Zakharov, Nucl. Phys. B147, 385 (1979).

[27] M. Gourdin and E. de Rafael, Nucl. Phys. B10, 667 (1969). 
[28] R. Alemany, M. Davier and A. Höcker, Eur. Phys.J. C2, 123 (1998).

[29] P. Duren, Theory of $H^{p}$ Spaces, (Academic Press, New York, 1970);

J. B. Garnett, Bounded Analytic Functions, (Academic Press, New York, 1981).

[30] F. Guerrero and A. Pich, Phys. Lett. B412, 382 (1997).

[31] G. Ecker, J. Gasser, A. Pich and E. de Rafael, Nucl. Phys. B321, 311 (1989).

[32] G. Ecker, J. Gasser, H. Leutwyler, A. Pich and E. de Rafael, Phys. Lett. B233, 425 (1989).

[33] R. Omnès, Nuovo Cim. 8, 316 (1958).

[34] I. Caprini and P. Dita, J.Phys.A:Math.Gen. 13, 1265 (1980).

[35] R. Barate et al (ALEPH Collaboration) Z. Phys. C76, 15 (1997).

[36] G.J. Gounaris and J.J.Sakurai, Phys. Rev. Lett. 21, 244 (1968).

[37] T. N. Truong, hep-ph/9809476.

[38] M. Davier, L. Girlanda, H. Höcher and J. Stern, Phys. Rev. D58; 096014 (1998).

[39] J. F. Donoghue and E. S. Na, Phys. Rev. D56, 7073 (1997).

[40] K.M. Watson, Phys. Rev. 95, 228 (1954).

[41] S.D. Protopopescu et al., Phys. Rev. D7, 1279 (1973).

P. Estabrooks and A.D. Martin, Nucl. Phys. B79, 301 (1974).

[42] J. Loewe, in [44], p. 368, and hep-ph/9711361.

[43] D. Počanić, in [44], p. 352, and hep-ph/9801366.

[44] Chiral Dynamics: Theory and Experiment, Proceedings of the Workshop held in Mainz, Germany, 1997 (Lecture Notes in Physics, Vol. 513, A. Bernstein, D. Drechsel and T. Walcher, Eds).

[45] C. Bourrely, Nucl. Phys. B43, 434 (1972).

[46] G. Auberson, Nucl. Phys.B98, 204 (1975).

[47] I. Caprini, Nuovo Cim. 49A, 307 (1979).

[48] I. Caprini, Phys. Rev. D27, 1479 (1983).

[49] N. I. Mushelishvili, Singular Integral Equations (Noordhoff, Groningen, 1953).

[50] D. G. Luenberger, Optimization by Vector Space Methods (Wiley, New York, 1969). 
[51] L.M. Barkov et al. (OLYA, CMD Collaboration), Nucl. Phys. B256, 365 (1985). D. Bisello et al. (DME Collaboration), Phys. Lett. B220, 321 (1989).

[52] M.G. Krein and P.I. Nudelman, Dokl.Acad. Nauk SSSR 209, 537 (1973).

[53] M. Rosenblum and J. Rovnyak, Proc. Amer. Math. Soc. 51, 335 (1975).

[54] S. Ciulli, C. Pomponiu and I. S. Stefanescu, Phys. Rep. C17, 133 (1975). 